\title{
Carcinoma anaplásico de tireoide: relato de caso tratado com cirurgia e radioterapia adjuvante
}

\author{
Anaplastic thyroid cancer: a case report treated with surgery and adjuvant \\ radiotherapy
}

Ana Clara Oliveira Tosta Telles ${ }^{1 *}$, Helton Estrela Ramos ${ }^{2}$

\begin{abstract}
${ }^{1}$ Mestranda do Programa de Pós-graduação Processos Interativos dos Órgãos e Sistemas. Universidade Federal da Bahia (UFBA); ${ }^{2 *}$ Professor Associado, Chefe do Laboratório de Estudo da Tireóide. Instituto de Ciências da Saúde da UFBA
\end{abstract}

\begin{abstract}
Resumo
Introdução: o Carcinoma Anaplásico de Tireoide (CAT) está entre as mais letais malignidades humanas, sendo a taxa de sobrevida estimada em 10-20\% em 01 ano e menor que $5 \%$ em 10 anos. Diante da raridade do CAT e desfecho consideravelmente desfavorável da doença, este relato discute as modalidades terapêuticas utilizadas no tratamento do CAT e as características da doença possivelmente relacionadas a um melhor desfecho clínico. Objetivo: relatar o caso de um paciente idoso portador de CAT com resposta completa loco-regional após tratamento combinado com cirurgia e radioterapia (RT) adjuvante. Neste estudo, a literatura a respeito das características da patologia da neoplasia indiferenciada da tireoide e modalidades de tratamento no controle oncológico desta doença é revisada e discutida. Caso clínico: trata-se de um paciente masculino de 88 anos submetido a Tireoidectomia Total (TT) cujo estudo imuno-histoquímico evidenciou neoplasia maligna indiferenciada da tireoide. O paciente realizou tratamento adjuvante com RT na dose total de $66 \mathrm{~Gy}$ em leito operatório. Em tempo de seguimento de 18 meses, o paciente encontra-se vivo sem doença detectável em atividade. Conclusão: neste relato, descreveu-se um raro caso de uma evolução favorável de um paciente idoso portador de CAT com longa sobrevida livre de doença quando comparada ao prognóstico reservado dessa neoplasia. Este relato destaca a importância de uma terapia multimodal no manejo desta doença.
\end{abstract}

Palavras-chave: Carcinoma anaplásico de tireoide. Tireoidectomia Total. Radioterapia.

\begin{abstract}
Backgroud: anaplastic thyroid cancer (ATC) ranks among the most lethal of all human malignancies, and the estimated survival rate ranges from 10 to $20 \%$ in 01 year and is less than $5 \%$ in 10 years. In view of the rarity of ATC and considerably unfavorable outcome of the disease, this report makes it possible to discuss the therapeutic modalities in the treatment of the ATC and the features of the disease possibly related to a better clinical outcome. Objective: the objective of the present study is to report the case of an elderly patient with ATC with locoregional complete response after combined treatment with surgery and adjuvant radiotherapy (RT.) In this study, the literature regarding the pathological features of the undifferentiated thyroid cancer and treatment modalities on oncologic outcome is reviewed and discussed. Case presentation: this is a case of a 88 year old male patient, who underwent total thyroidectomy (TT) for thyroid cancer treatment whose cytological analysis was compatible with anaplastic thyroid cancer. The immunohistochemical study showed undifferentiated malignancy of the thyroid. The patient underwent adjuvant treatment with RT at the total dose of 66 Gy in operative bed. In a follow-up period of 18 months, the patient is alive with no detectable disease in activity. Conclusions: in this report, is described a rare case of a favorable evolution of an elderly patient with ATC relatively long disease-free survival compared to the reserved prognosis of this neoplasm. This case underlines the importance of a multimodal therapy in the management of this disease.
\end{abstract}

Key words: Anaplastic Thyroid Cancer. Total thyroidectomy. Radiotherapy

\section{INTRODUÇÃO}

O carcinoma anaplásico da tireóide (CAT) está entre as mais letais malignidades humanas ${ }^{1}$. Apesar de corresponder apenas a $2 \%$ de todas as neoplasias de tireóide, é a causa de morte da metade dos pacientes, nos quais a causa definitiva da morte é o câncer tireoidiano ${ }^{2}$. A sobrevida é mensurada em meses e são tumores caracterizados

Correspondente/Corresponding: *Ana Clara Oliveira Tosta Telles - Instituto de Ciências da Saúde - Universidade Federal da Bahia - End: Av. Reitor Miguel Calmon, S/N; Vale do Canela, Salvador - BA - CEP: 40.110902. - Tel. (71)99114-4445 - E-mail: ana.telles@ufba.br por altas taxas de crescimento local e disseminação metastática à distância ${ }^{1}$, sendo a taxa de sobrevida estimada em $10-20 \%$ em 1 ano e menor que $5 \%$ em 10 anos $^{3}$.

A maioria dos pacientes afetados tem mais de 50 anos de idade, com um pico de incidência na 6a e $7 \underline{\text { a }}$ décadas de vida e com proporção entre o sexo feminino e masculino na razão $1,5: 2^{4}$. O sexo masculino está associado a um pior prognóstico no CAT, com sobrevida livre de doença mais curta e maior mortalidade ${ }^{5}$. Pode-se afirmar que a incidência e a taxa de mortalidade do CAT têm se mantido relativamente estável, e não houve melhora do desfecho oncológico nos últimos 30 anos. Por outro lado, uma van- 
tagem na sobrevida tem sido demonstrada em jovens e pacientes sem metástase à distância ${ }^{6}$.

Devido ao prognóstico pobre, pacientes com CAT são tipicamente tratados com uma combinação de cirurgia, terapia sistêmica e radioterapia (RT) ${ }^{7}$, os quais, parecem resultar em aumento de sobrevida em paciente com estadiamento clínico IVA/B $B^{8}$. Em pacientes tireoidectomizados, o aumento da sobrevida se relaciona à presença de doença ressecável; inversamente, pacientes que não recebem tratamento cirúrgico tendem a se apresentar com doença avançada ${ }^{9}$. Dessa forma, é possível explorar intervenções mais agressivas em pacientes com doença precoce, intra-tireoidiana ou com extensão extratireoidiana mínima, que possuem o melhor prognóstico e são candidatos a ressecções curativas (R0, margens cirúrgicas negativas) ${ }^{10}$.

Neste artigo, é relatado um caso de um paciente idoso portador de CAT de rápida progressão atendido no Hospital Aristides Maltez, submetido a tratamento combinado com tireoidectomia total (TT) e RT adjuvante, assim como, o desfecho oncológico do paciente com satisfatório controle loco-regional após um período de seguimento relativamente longo de 18 meses, haja vista o prognóstico reservado e a baixa taxa de sobrevida dos pacientes portadores desta doença. Além disso, este texto revisa a literatura a respeito das características patológicas da neoplasia indiferenciada da tireóide e discute as modalidades de tratamento e seu impacto no controle oncológico desta doença.

\section{CASO CLÍNICO}

Um homem idoso de 88 anos de idade foi referenciado ao Hospital Aristides Maltez em janeiro de 2019 após um período de 4 meses de aumento de volume da região cervical anterior de rápido crescimento. Quando da admissão no serviço de cirurgia de cabeça e pescoço da instituição, o paciente queixava-se de tosse seca, sem demais queixas relacionadas ao trato aero digestivo superior, tais como disfagia, odinofagia, estridor ou dispneia. Negou hábitos de tabagismo e etilismo durante a vida; negou ser portador de quaisquer comorbidades conhecidas, assim como não fazia uso crônico de medicamentos. Nenhuma doença tireoidiana benigna concomitante foi reportada.

Acrescenta-se que não havia história pregressa de irradiação de região de cabeça e pescoço. Tampouco havia histórico familiar de câncer e doenças da tireoide em parentes de primeiro grau. À consulta clínica inicial, o paciente apresentava-se fisicamente bem e capaz de desempenhar suas atividades habituais sem restrição, apesar da idade.

Portanto, de acordo com a escala Eastern Cooperative Oncology Group (ECOG) para performance status, ele foi classificado como ECOG grau 0. Ao primeiro exame físico, um nódulo bem palpável em topografia de lobo direito da tireoide foi detectado com aproximadamente $4,5 \mathrm{~cm}$, endurecido, indolor e pouco móvel à deglutição, sem linfonodos cervicais palpáveis bilateralmente no pescoço (Figura 1). As pregas vocais estavam móveis à laringoscopia.

Figura 1 - Tumoração em loja tireodiana à direita expansiva com compressão local da traqueia, abaulando a região cervical anterior.

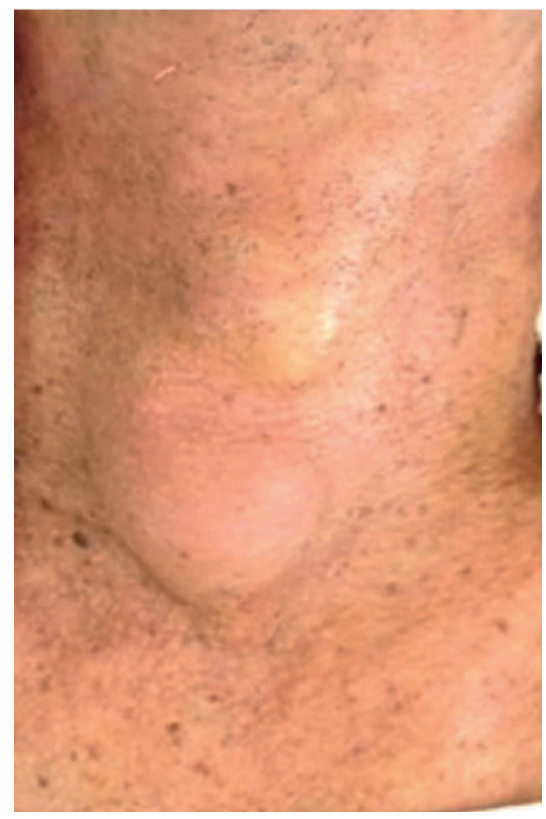

Fonte: Paciente caso clínico

Clinicamente, não havia sinais de estridor laríngeo ou de obstrução da via aérea superior. O paciente já havia realizado previamente uma Punção Aspirativa por Agulha Fina (PAAF) desta tumoração guiada por ultrassonografia, a qual revelava um nódulo sólido em istmo/lobo direito da tireoide medindo $4,0 \times 2,5 \times 3,8 \mathrm{~cm}$ e um nódulo em lobo esquerdo medindo $0,4 \times 0,4 \mathrm{~cm}$, com calcificações grosseiras cuja citologia oncótica foi compatível com Carcinoma Anaplásico da tireóide em lobo direito.

A partir daí o paciente foi encaminhado para estadiamento tumoral imagenológico com Tomografia Computadorizada (TC) do pescoço e do tórax com contraste, sendo evidenciado um nódulo de $4.6 \times 4,2 \times 2,8 \mathrm{~cm}$ (diâmetros Lateral $x$ Antero-posterior $X$ Transverso) em lobo direito da tireoide com calcificação grosseira e realce heterógeno após o contraste, com áreas hipodensas sugestivas de degeneração cística ou necrose.

À TC, a tumoração apresentava ainda sinais de invasão extra-tireoidiana (musculatura pré-tireoidiana), abaulando o contorno anterior da pele da região cervical anterior, se estendendo ao intróito torácico, e promovendo desvio contralateral da traqueia ao nível do mediastino superior. Não foram identificadas linfonodomegalias cervicais (Figura 2). A TC de tórax afastou metástase pulmonar, sem demais achados adicionais. Após o estadiamento de imagem, o paciente foi julgado clinicamente ressecável, sendo encaminhado para tratamento cirúrgico com TT em 18/01/19, no mesmo mês de sua admissão na instituição. 
Figura 2 - TC de pescoço com contraste: tumor em lobo direito da tireóide com calcificação grosseira e realce heterogêneo após o contraste, com áreas hipodensas sugestivas de degeneração cística ou necrose, sinais de invasão extra-tireoidiana com desvio contralateral da traqueia ao nível do mediastino superior.
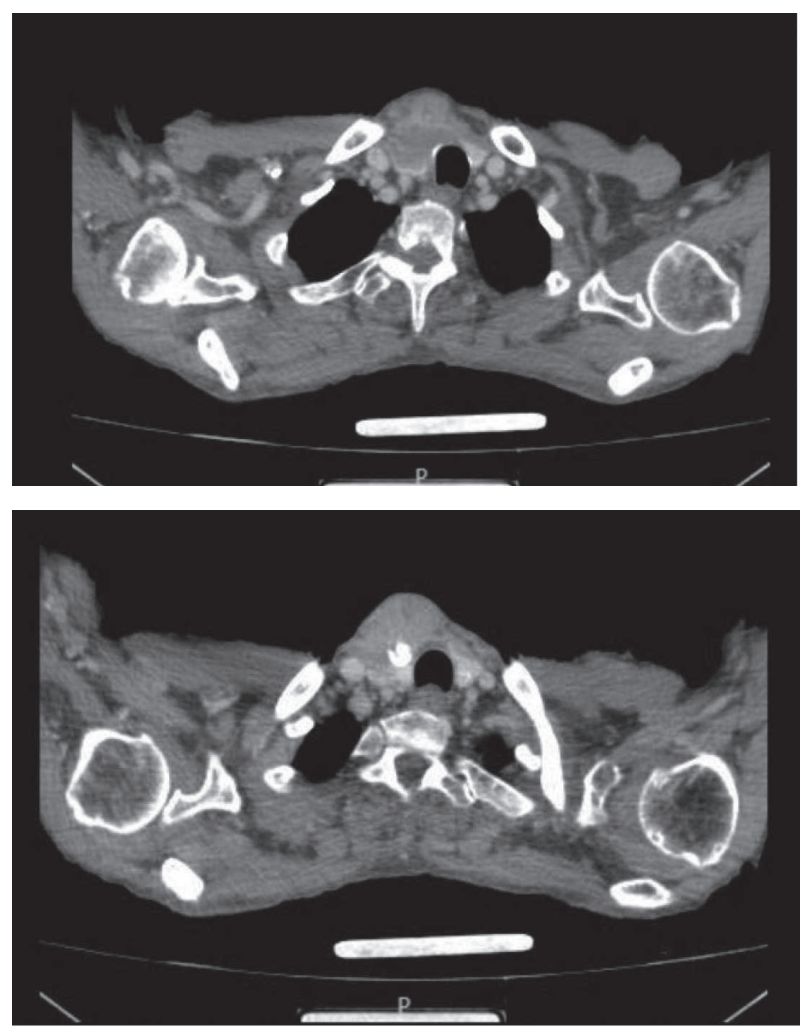

Fonte: Paciente caso clínico

No intra-operatório, foi identificada uma tumoração em lobo direito em transição com istmo da tireóide de aproximadamente $5 \mathrm{~cm}$ com sinais de extensão extra-capsular da glândula, envolvendo a musculatura pré-tireoidiana, e acometimento tumoral superficial da parede traqueal anterior ao nível do seu segundo e terceiro anéis traqueais, sem infiltração de sua luz. Realizada TT com ressecção em bloco com parte do músculo esterno-tiróideo com a peça cirúrgica (Figura 3).

Figura 3- Peça cirúrgica evidenciando tumoração irregular e volumosa em lobo direito e istmo da tireoide.

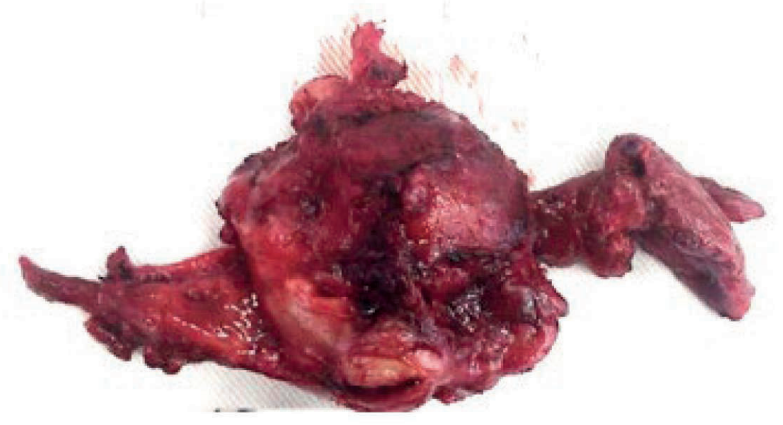

Fonte: Paciente caso clínico
Além disso, foi optado por realizar "shaving" de traqueia, sem ressecção de anel traqueal com o intuito de evitar agregar complicações cirúrgicas, considerando a idade avançada do paciente. À exploração cervical do nível VI no intra-operatório, após a excisão da peça cirúrgica, não foram identificados linfonodos de aspecto patológico, não sendo indicado, portanto, o esvaziamento cervical deste compartimento.

O estudo anatomopatológico confirmou uma neoplasia maligna de lobo direito da tireóide medindo 4,0 x $2,2 \mathrm{~cm}$ com extensão extra-capsular, com margens cirúrgicas comprometidas pela neoplasia, porém sem invasão microscópica de tecido extra-tireoidiano. Nesta primeira análise, não foi completamente afastado carcinoma, mas a dosagem das citoqueratinas foi negativa, o que pode ocorrer na neoplasia indiferenciada da tireóide.

Nesse respeito não foi definido o tipo histológico da neoplasia maligna, sendo solicitada ampliação de painel de anticorpos. A análise imuno-histoquímica evidenciou uma neoplasia maligna indiferenciada negativa para marcadores epiteliais assim como para marcadores de doença linfoproliferativa e melanoma, aventados como diagnóstico diferencial no estudo patológico.

Posteriormente, realizada ampliação de painel para afastar sarcoma, fortalecendo o diagnóstico de carcinoma indiferenciado da tireoide (Figura 4). O estadiamento final da neoplasia foi então definido como T4aNOMO, estágio IVB, de acordo com o TNM Classification of malignat tumors ${ }^{14}$.

Figura 4- Estudo anatomopatológico e análise imunohistoquímica favoreceram o diagnóstico de carcinoma indiferenciado da tireoide
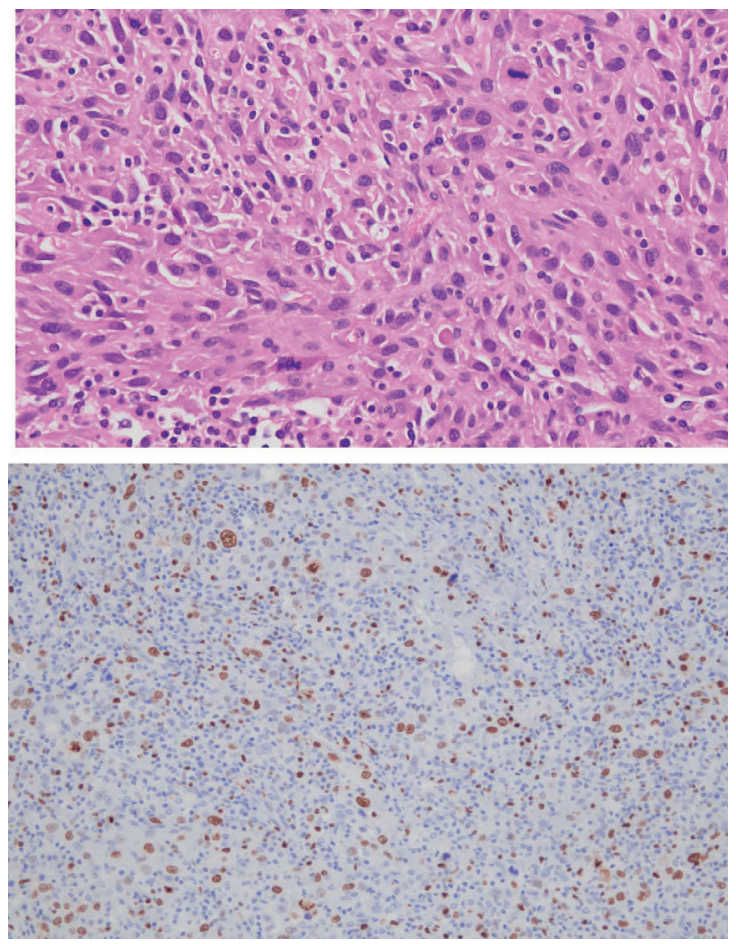

Fonte: Paciente caso clínico 
Não houve complicações clínicas ou cirúrgicas. Levotiroxina e carbonato de cálcio foram administrados no pós-operatório. No seguimento clínico, a dosagem de cálcio e paratormônio séricos se mantiveram dentro dos limites de normalidade e não houve necessidade de administração de cálcio continuamente.

$\mathrm{Na}$ primeira consulta pós-operatória foi realizada laringoscopia, sem evidência de alteração de mobilidade das pregas vocais. Exame tomográfico do pescoço de controle realizado em abril/2019 não evidenciou sinais de doença residual. O paciente foi encaminhado para tratamento adjuvante com RT em função do estadiamento tumoral e comprometimento das margens cirúrgicas; e não indicada quimioterapia adjuvante em razão dos riscos de o tratamento serem possivelmente superiores aos benefícios relacionados a este caso e, em especial, da idade avançada do paciente.

O paciente foi submetido a RT adjuvante conformacional em 3D em leito cirúrgico com dose total de $66 \mathrm{~Gy}$ com fracionamento convencional em 33 frações no período de maio a julho 2019, com o objetivo de reduzir o risco de recorrência local. Conforme a escala de toxicidade Common Toxicity Criterio - CTC, ocorreram mucosite grau 1 e radiodermite grau 2 no curso do tratamento, ambos efeitos adversos bem tolerados com uso de corticosteroide oral e terapia tópica local (Figura 5).

Figura 5 - Aspecto clínico do pescoço pós-tratamento com radioterapia adjuvante.

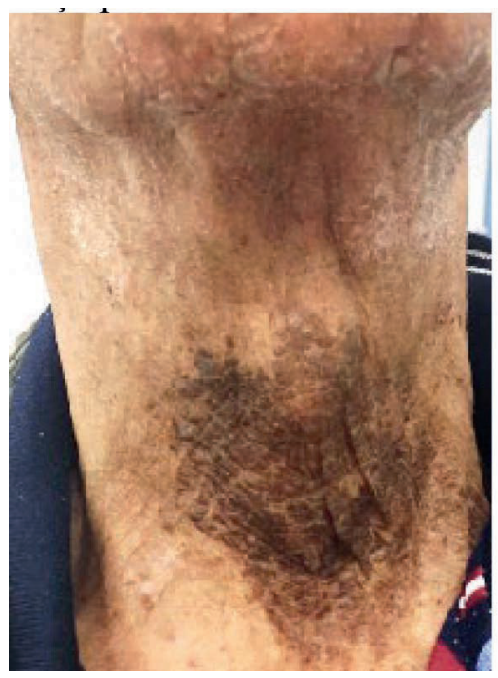

Fonte: Paciente caso clínico

O tratamento não teve impacto constitucional no paciente, apesar dos sintomas de leve inapetência e odinofagia relatados durante a RT. Desde o término do tratamento, o paciente manteve-se bem clinicamente e assintomático, seguindo as recomendações de acompanhamento regular no serviço de cirurgia de cabeça e pescoço a cada 3 meses.
A avaliação endocrinológica incluindo prova de função tireoidiana foi requerida em cada visita clínica. O USG do pescoço e tomografia computadorizada de pescoço, tórax e abdome com contraste foram realizados após 4 meses do término da RT. Após 6 meses do último exame de imagem, repetiu-se o estudo tomográfico de pescoço e tórax, todos sem evidência de doença loco-regional ou à distância.

\section{DISCUSSÃO}

O CAT é a forma de neoplasia tireodiana com o pior prognóstico e é uma entidade bastante rara. Sua etiologia é desconhecida ${ }^{2}$, correspondendo a apenas $1-2 \%$ das neoplasias da tireoide. A maioria dos pacientes têm 65 anos de idade ou mais ao diagnóstico e apresentam massa cervical de rápido crescimento, disfagia e alteração da $v z^{4}$. Idade avançada, estadiamento tumoral e tamanho do tumor têm se configurado como fatores prognósticos negativos na sobrevida desses pacientes ${ }^{5}$.

O estudo do Anaplastic Thyroid Carcinoma Research Consortium of Japan através da análise de 68 pacientes com sobrevida longa (superior a 1 ano) sugeriu que a presença de um tumor tireodiano pré-existente, infiltração linfocítica e não neutrofílica, componente escamoso e crescimento epitelial do tumor podem estar associados a melhor prognóstico no $\mathrm{ACT}^{11}$. A neoplasia do paciente mencionado apresentava à imuno-histoquímica marcadores epiteliais (citoqueratinas AE1/AE3) negativos, mas apresentava infiltrado de linfócitos e macrófagos, o que pode ter contribuído para um melhor prognóstico relacionado à essa característica anatomopatológica específica.

O impacto do volume tumoral assim como os critérios anatomopatológicos foram também analisados por Oh et al. ${ }^{12}$ e relacionados ao tempo de sobrevida global. Todos os pacientes que sobreviveram por mais de 6 meses foram submetidos a TT e possuíam tumores com tamanho menor de $5 \mathrm{~cm}$, sem invasão linfática e eram mais jovens ao diagnóstico, com média de idade de 55 anos $^{12}$. Assim, considerou-se que pacientes com tumores de menor volume e sem doença metastática são bons candidatos a tratamento cirúrgico.

O paciente deste estudo apresentava uma tumoração medindo $4,0 \mathrm{~cm}$ em maior diâmetro no estudo anatomopatológico, que foi ressecada com a TT com êxito, a despeito das margens positivas à microscopia, o que caracteriza um perfil de paciente elegível a tratamento com intenção curativa.

O papel da cirurgia no manejo dos pacientes com CAT é bastante discutido porque está associado às características da doença, com a maioria desses tumores se apresentando com um estadiamento muito avançado ao diagnóstico, o que pode implicar em ressecção de estruturas vitais e impedir o tratamento curativo.

Um grande estudo multicêntrico coreano publicado pelo Korean Society of thyroid of head and neck surgery avaliou os fatores prognósticos e modalidades de tra- 
tamento de 329 pacientes de 19 instituições, a maioria deles, estadiamento IVB (49,5\%). Dentre as características clínicas, a idade $>70$ anos e estadiamento avançado da doença (com metástases à apresentação) estavam relacionados a um pior prognóstico, com impacto na sobrevida. A TT foi realizada em $60,2 \%$ dos pacientes e a melhor taxa de sobrevida global em 1 ano $(50,2 \%)$ foi observada no grupo tratado com cirurgia curativa e RT adjuvante, ou cirurgia e RT com quimioterapia concomitante adjuvante, não havendo comparação entre os dois grupos de tratamento mencionados ${ }^{13}$.

Neste grande estudo multicêntrico, a idade acima de 70 anos foi um fator de pior prognóstico. A idade isoladamente do paciente supracitado não se demonstrou como um fator prognóstico importante, devendo-se levar em consideração os demais fatores associados, como estadiamento tumoral, como bem demonstrado por este estudo.

De acordo com o TNM Classification of malignat tumors, no estadiamento IVB, já há extensão extra-tiroidiana da neoplasia, mas sem doença metastática à distância. No estágio IVA, a doença está localizada na glândula tireoide; e, o estágio IVC representa os tumores com doença extra-tireodiana irressecável localmente, e os tumores que já se apresentam com metástase à distância ao diagnóstico ${ }^{14}$.

O estadiamento patológico final do caso deste artigo foi IVB em razão da extensão tumoral extra-tireoidiana ao anel traqueal, ainda que de pequena monta. Brown et al. ${ }^{15}$ avaliaram 38 pacientes com CAT operados, todos com extensão extra-tireoidiana do tumor, em um período de 14 anos. Destes, 16 pacientes foram tratados com ressecção completa da doença macroscópica, dos quais, 14 pacientes receberam RT adjuvante com dose de 50 a 70 Gy e nenhum recebeu quimioterapia concomitante. Dos pacientes tratados com ressecção completa e RT adjuvante, nenhum apresentou recorrência local ${ }^{15}$. Esses estudos sugerem que o tratamento multimodal pode ser aplicado nos pacientes com doença com extensão extra-glandular com aumento de sobrevida global e livre de recorrência local.

Devido a esses estudos serem limitados, com pequenas amostras, sendo a maioria deles retrospectivos e heterogêneos a respeito da RT no tratamento do CAT, o melhor regime de dose total e fracionamento ainda precisa ser esclarecido. Assim, a atuação da RT nos pacientes portadores de CAT é criticada em relação ao controle loco-regional da doença porque a mortalidade geralmente é secundária a complicações relacionadas ao volume tumoral no sítio primário da doença.

Fan et al. ${ }^{7}$ avaliaram 104 pacientes de uma única instituição com CAT admitidos em um período de 33 anos que receberam tratamento com intenção curativa. Destes os pacientes, $73,1 \%$ receberam terapia trimodal (cirurgia, RT e quimioterapia adjuvantes), com dose média de 66 Gy. A dose de RT $\geq 60 \mathrm{~Gy}$ foi associada ao menor risco de progressão local e a sobrevida global média desses pacientes foi de 10,6 meses comparada a 3,6 meses daqueles pacientes que receberam dose $<60 \mathrm{~Gy}$, sugerindo que doses altas de RT podem ser efetivas em pacientes selecionados ${ }^{7}$.

O paciente nesse estudo de caso recebeu tratamento com RT adjuvante com alta dose de 66Gy em leito operatório com intenção curativa, de acordo com a intenção do procedimento cirúrgico proposto de TT, sem evidência de recorrência loco-regional no seguimento clínico.

O regime de RT e sua toxicidade no tratamento do CAT foi avaliado por Corrigan et al. ${ }^{16}$ quanto à dose menor ou maior do que $40 \mathrm{~Gy}$, com a utilização da RT conformacional (2D e 3D) e da RT de intensidade modulada (IMRT). Apesar de a literatura apontar um aumento na sobrevida com maiores doses de $R T^{7,15}$, este estudo não demonstrou esse benefício com o aumento da dose acima de 40Gy ou com o hiperfracionamento da dose. Foi observado também que os pacientes tratados com IMRT experimentaram menos toxicidade, porém sem diferenças significantes em relação ao grupo tratado com RT conformacional ${ }^{16}$.

O paciente em questão foi submetido a RT conformacional 3D porque a instituição onde foi tratado não dispõe de IMRT, contudo, ele apresentou toxicidade bem tolerada com mucosite grau 1 e radiodermite grau 2 , que não foram suficientes para interrupção do tratamento, sem impacto importante na sua performance status.

A primeira metanálise com 17 estudos que avaliou o impacto da RT pós-operatória nos pacientes operados de CAT por qualquer tipo de ressecção cirúrgica demonstrou que a RT adjuvante reduziu significativamente o risco de morte de todos os pacientes operados quando comparada ao grupo submetido a cirurgia isolada, para os pacientes com estadiamento IVA e IVB, exceto pelos pacientes IVC ${ }^{17}$; e o tratamento com RT e/ou quimioterapia combinado à cirurgia pareceu aumentar a sobrevida nesses pacientes ${ }^{8}$.

Outra metanálise com 10 estudos relevantes questionou se a quimioterapia pós-operatória também pode prolongar a sobrevida dos pacientes com CAT, mas concluiu que não houve ganho de sobrevida, diferentemente da RT adjuvante nesses $\operatorname{casos}^{18}$; já o estudo de Lee et al. ${ }^{19}$ sugere que os pacientes submetidos a um tratamento de maior intensidade com RT e quimioterapia adjuvantes têm uma tendência a apresentar maior sobrevida ${ }^{19}$.

Saeed et al. ${ }^{20}$ analisaram 496 pacientes do National Cancer Database com CAT não metastático submetidos a ressecção cirúrgica não paliativa, e recomendam que a quimioterapia concomitante pode ser administrada como agente radiossensibilizante com o objetivo de controle local, ainda que não haja uma diretriz a respeito da quimioterapia em relação aos CAT ressecados ${ }^{20}$. Como a quimioterapia não tem demonstrado um benefício significante na sobrevida em muitos desses estudos e pode implicar em maior morbidade em alguns pacientes, especialmente em doentes mais idosos, decidiu-se não oferecer essa modalidade de tratamento ao paciente deste estudo.

Esta breve revisão de literatura aponta que o tratamento inicial recomendado é a ressecção cirúrgica e que a RT adjuvante pode aumentar a sobrevida desses 
pacientes em doenças ressecáveis, portanto, a indicação do tratamento cirúrgico depende fundamentalmente das condições clínicas do paciente e da extensão da doença ao diagnóstico, desde que não apresentem doença metastática à distância na avaliação pré-operatória.

\section{CONCLUSÃO}

O prognóstico dos pacientes com CAT tratados com terapia combinada ainda é pobre, portanto, ao considerar o estudo deste relato e a partir da revisão da literatura a respeito das modalidades de tratamento do CAT acredita-se que a ressecção cirúrgica completa em pacientes portadores de CAT selecionados associada a RT adjuvante é capaz de oferecer uma razoável sobrevida a esses pacientes e com melhor controle loco-regional da doença. Infelizmente, o CAT é pouco responsivo à quimioterapia, mas terapias alvo e imunoterapia a nível molecular podem trazer novas possibilidades de opções de tratamento sistêmico para esses doentes.

\section{REFERÊNCIAS}

1. WISEMAN, S. M. et al. Anaplastic transformation of thyroid cancer: review of clinical, pathologic, and molecular evidence provides new insights into disease biology and future therapy. Head Neck, New York, v. 25, n.8, p. 662-670, 2003

2. ZIVALJEVIC, V. et al. Risk factors for anaplastic thyroid cancer. Int. J. Endocrinol., [s.I], 2014.

3. NAGAIAH, G. et al. Anaplastic thyroid cancer: a review of epidemiology, pathogenesis, and treatment. J. Oncol.[s.I], 2011.

4. MOLINARO, E. et al. Anaplastic thyroid carcinoma: from clinicopathology to genetics and advanced therapies. Nat. Rev. Endocrinol., London, v.13, n.11, p.644-660, 2017.

5. RAO, S. N. et al. Patterns of treatment failure in anaplastic thyroid carcinoma. Thyroid. New York, v.27, n.5, p.672-681, 2017.

6. LIN, B. et al. The incidence and survival analysis for anaplastic thyroid cancer: a SEER database analysis. Am. J. Transl. Res, [s.I], v.11, n.9, p.5888-5896, 2019.

7. FAN, D. et al. Outcomes of multimodal therapy in a large series of patients with anaplastic thyroid cancer. Cancer, London, v.126, n.22, p.444-452, 2020.

8. WÄCHTER, S. et al. Anaplastic thyroid carcinoma: changing trends of treatment strategies and associated overall survival. Eur. Arch. Otorhinolaryngol., Germany, v.277, p.1507-1514, 2020.

9. JANZ, T. A. et al. Is the incidence of anaplastic thyroid cancer increasing: A population based epidemiology study. World J. Otorhinolaryngol. Head Neck Surg., Beijing, v.5, n.1, p.34-40, 2018.

10. HU, S. et al. The role of surgery in anaplastic thyroid cancer: a systematic review. Am. J. Otolaryngol., Philadelphia, v.38. n.3, p.337350, 2017.

11. HIROKAWA, M. et al. Histopathological analysis of anaplastic thyroid carcinoma cases with long-term survival: areport from the Anaplastic thyroid carcinoma research consortium of Japan. Endocr. J., Tokyo, v. 63, n.5, p.441-447, May 2016.

12. OH, E. M. et al. Analysis of patients with anaplastic thyroid cancer expected to have curative surgery. J. Korean Surg. Soc., Korea, v.83, n.3, p.123-129, Sept. 2012.

13. BAEK, S. K. et al. Role of surgery in the management of anaplastic thyroid carcinoma: Korean nationwide multicenter study of 329 patients with anaplastic thyroid carcinoma, 2000 to 2012. Head Neck, New York, v.39, n.1, p.133-139, jan. 2017.

14. BRIERLEY, J. D.; GOSPODAROWICZ, M. K.; WITTEKIND, C. (Eds.). TNM Classification of malignat tumors. 8. ed. [s.I: s,n], 2017.

15. BROWN, R. F.; DUCIC, Y. Aggressive surgical resection of anaplastic thyroid carcinoma may provide long-term survival in selected patients. Otolaryngol. Head Neck Surg., Rochester, v.148, n.4, p.564-571, Apr. 2013.

16. CORRIGAN, K. L, et al. Treatment outcomes in anaplastic thyroid cancer. J Thyroid Res., [s.I], v.2019, p.8218949, May 2019.

17. KWON, J. et al. The prognostic impacts of postoperative radiotherapy in the patients with resected anaplastic thyroid carcinoma: a systematic review and meta-analysis. Eur. J. Cancer, Oxford, v.59, p.34-45, May 2016.

18. XIA, Q. et al. Evidence from an updated meta-analysis of the prognostic impacts of postoperative radiotherapy and chemotherapy in patients with anaplastic thyroid carcinoma. Onco Targets Ther., [s.I], v.11, p.2251-2257, Apr. 2018.

19. LEE, J. H. et al. Optimal combination of treatment modality to increase survival in patients with anaplastic thyroid carcinoma: a STROBE compliant retrospective study. Medicine (Baltimore), Baltimore, v.97, n.25, p.11037, June 2018.

20. SAEED, N. A. et al. Adjuvant external beam radiotherapy for surgically resected, nonmetastatic anaplastic thyroid cancer. Head Neck, New York, v.42, n.5, p.1031-1044, May 2020.

Submetido em: $07 / 12 / 2020$

Aceito em: 14/12/2020 